\title{
A heterozygous variant in the human cardiac miR-133 gene, MIR133A2, alters miRNA duplex processing and strand abundance
}

\author{
Monique Ohanian ${ }^{1+}$, David T Humphreys ${ }^{2,3+}$, Elizabeth Anderson ${ }^{4}$, Thomas Preiss ${ }^{5}$ and Diane Fatkin ${ }^{1,2,3,6^{*}}$
}

\begin{abstract}
Background: MicroRNAs (miRNAs) are small non-coding RNAs that post-transcriptionally regulate gene expression. Sequential cleavage of miRNA precursors results in a 22 nucleotide duplex of which one strand, the mature miRNA, is typically loaded into the RNA-induced silencing complex (RISC) while the passenger strand is degraded. Very little is known about how genetic variation might affect miRNA biogenesis and function.

Results: We re-sequenced the MIR1-1, MIR1-2, MIR133A1, MIR133A2, and MIR133B genes, that encode the cardiacenriched miRNAs, miR-1 and miR-133, in 120 individuals with familial atrial fibrillation and identified 10 variants, including a novel 79T > C MIR133A2 substitution. This variant lies within the duplex at the $3^{\prime}$ end of the mature strand, miR-133a-3p, and is predicted to prevent base-pairing and weaken thermostability at this site, favoring incorporation of the passenger strand, miR-133a-5p, into RISC. Genomic DNA fragments containing miR-133a-2 precursor sequences with $79 \mathrm{~T}$ and $79 \mathrm{C}$ alleles were transfected into HeLa cells. On Northern blotting the 79T allele showed strong expression of miR-133a-3p with weak expression of miR-133a-5p. In contrast, the 79C allele had no effect on miR-133a-3p but there was a significant increase (mean 3.6-fold) in miR-133a-5p levels. Deep sequencing of small RNA libraries prepared from normal human and murine atria confirmed that nearly all the mature miR-133a was comprised of miR-133a-3p and that levels of miR-133a-5p were very low. A number of isomiRs with variations at $5^{\prime}$ and $3^{\prime}$ ends were identified for both miR-133a-3p and miR-133a-5p, with 2 predominant miR-133a-3p isomiRs and one predominant miR-133a-5p isomiR. Bioinformatics analyses indicate that the major miR-133a-3p and $5 p$ isomiRs have numerous predicted target mRNAs, only a few of which are in common.

Conclusions: Multiple miR-133a isomiRs with potential different mRNA target profiles are present in the atrium in humans and mice. We identified a human 79T > C MIR133A2 variant that alters miRNA processing and results in accumulation of the miR-133a-5p strand that is usually degraded.
\end{abstract}

Keywords: MicroRNA, isomiR, Genetics, Atrial fibrillation

\section{Background}

MicroRNAs (miRNAs) are small non-coding RNAs that regulate gene expression. They act by binding to the 3 'untranslated regions of target mRNAs and typically reduce mRNA levels by inhibiting translation or stimulating mRNA decay [1]. MiRBase v18 [2] lists 2148 human

\footnotetext{
* Correspondence: d.fatkin@victorchang.edu.au

${ }^{\dagger}$ Equal contributors

${ }^{1}$ Molecular Cardiology Division, Victor Chang Cardiac Research Institute, Darlinghurst, New South Wales, Australia

${ }^{2}$ Molecular Genetics Division, Victor Chang Cardiac Research Institute, Darlinghurst, New South Wales, Australia

Full list of author information is available at the end of the article
}

miRNAs, each of which is expected to have multiple mRNA targets. Consequently, miRNAs can orchestrate suites of changes in gene expression and have been implicated in diverse normal physiological processes and disease states [3].

Most miRNAs are transcribed from their own genes by RNA polymerase II. A minority of miRNAs are located within introns of other genes and are cotranscribed with the host gene. In the canonical miRNA biosynthesis pathway, primary miRNA (pri-miRNA) hairpins are cleaved by the nuclear RNAase III-type endonuclease Drosha to yield a $\sim 70$ nucleotide (nt) precursor miRNA (pre-miRNA) that is then exported to the 
cytoplasm. Further cleavage of this stem-loop structure y Dicer generates $\mathrm{a} \sim 22$ nt mature miRNA duplex [4]. Typically, the dominant (mature) strand of the duplex is incorporated into an RNA-induced silencing complex (RISC) that binds to the target mRNA by sequencespecific interactions between the "seed" region (nt 2-8) at the $5^{\prime}$ end of the miRNA and the cognate mRNA binding site. The remaining (passenger) strand of the duplex is thought to be non-functional and degraded. Recent deep sequencing data have revealed surprising additional complexity of miRNA processing and have found that multiple miRNA isoforms (isomiRs) of varying sequence composition and length may be present [5-8].

The muscle-enriched miRNAs, miR-1 and miR-133, are amongst the most abundant of the miRNAs present in the normal heart $[9,10]$. Two genes, MIR1-1 and MIR1-2, encode miR-1-1 and miR-1-2, while three genes, MIR133A1, MIR133A2, and MIR133B, encode miR-133a-1, miR-133a-2, and miR-133b, respectively. MiR-133a-1 and miR-133a-2 have identical mature sequences, with miR-133b differing only by a single nt at the $3^{\prime}$ end. In humans, MIR1-1 and MIR133A2 are located on chromosome 20 while MIR1-2 and MIR133A1 are located on chromosome 18. These 2 pairs of genes are co-regulated and expressed as bicistronic transcripts. $M I R 133 B$ is on chromosome 6 and is paired with another muscle-specific miRNA gene, MIR206. Verified mRNA targets of miR-1 and miR-133 include those encoding proteins that are involved in cardiac development, ion channel function, hypertrophy, and fibrosis [11-16].

Atrial fibrillation (AF) is the most common cardiac arrhythmia and a frequent complication of diverse cardiac and systemic disorders. Altered levels of miR-1 and miR-133 have been observed in atrial tissue samples from patients with AF in several studies [17-19]. MiRNA-induced gene expression changes in the atrium could either have a primary role in initiation of AF or contribute to electrical and structural remodelling that perpetuates AF. In this study, we hypothesized that genetic variation could alter the functional effects of miR-1 and miR-133 and contribute to AF pathogenesis. To test this hypothesis, we performed genetic screening of the MIR1-1, MIR1-2, MIR133A1, MIR133A2, and MIR133B genes in a cohort of probands with suspected familial AF. A number of variants were identified, including a novel MIR133A2 variant that was functionally characterized and found to alter miR-133a duplex processing. To assess the potential effects of this variant, we first needed to catalogue the abundance and diversity of miR-133a isomiRs in the normal heart. Deep sequencing of human and murine atrial tissue was performed and revealed an unexpected diversity of miR-133a isomiRs, with nearly all the miR-133a tags comprised of the 2 major miR-133a-3p isomiRs and
$<1 \%$ comprised of miR-133a-5p species. Our data suggest that the MIR133A2 variant increases the relative abundance of miR-133a-5p.

\section{Results}

MiR-1 and miR-133 sequence variants

The 5 loci encoding miR-1 and miR-133 precursor transcripts were re-sequenced in 120 probands with a family history of AF. Ten variants were identified, 2 of which were novel (Table 1). For the 8 known variants, the minor allele frequencies in the AF group were similar to those reported in the public databases, dbSNP, NHLBI Exome Variant Server and 1000 Genomes. Three MIR133A2 variants, $-102 \mathrm{G}>\mathrm{A},-82 \mathrm{G}>\mathrm{A}$, and $-19 \mathrm{G}>\mathrm{A}$, were in linkage disequilibrium as reported previously [20]. A MIR133A2 haplotype that included $-102 \mathrm{G}>\mathrm{A},-82 \mathrm{G}>\mathrm{A}$, $-19 \mathrm{G}>\mathrm{A}$, and $+47 \mathrm{~T}>\mathrm{C}$ was present on one allele in $18 \mathrm{AF}$ probands (15\%) and 37 in-house controls (15\%), and on both alleles in $8 \mathrm{AF}$ probands (7\%) and 12 in-house controls (5\%). Of the 2 novel variants, only one was located within a miRNA stem-loop sequence. This variant, a 79T $>\mathrm{C}$ substitution in the MIR133A2 gene (Figure 1A), was found in a 67-year old female (II-5, Family KB, Figure 1B) who had paroxysmal episodes of AF, hypertension and mitral valve disease. The two other living family members with AF, II-1 and II-4, were genotype-negative. The $79 \mathrm{~T}>\mathrm{C}$ variant was not seen in clinically unaffected relatives, in our 250 healthy control subjects, or in the human genome sequence databases.

\section{T > C MIR133A2 variant increases levels of miR-133a-5p} The 79T > C MIR133A2 variant is positioned directly adjacent to the Drosha cleavage site in the stem-loop structure at the $3^{\prime}$ end of miR-133a-3p (Figure 1C). In the absence of sequence data for human miR-133a-5p in miRBase, this location was initially deduced from $3 p$ dominant processed sequence listed for mouse. We predicted that the mismatch introduced by the $79 \mathrm{~T}>\mathrm{C}$ substitution would alter secondary structure and thus precursor processing and/or subsequent strand selection. To test these predictions, we prepared two constructs that replicated the $79 \mathrm{~T}$ and $79 \mathrm{C}$ genotypes of mature miR-133a, transfected these into the HeLa cell line that does not detectably express endogenous miR-133a and performed Northern blotting. For these studies, the miR-133a-2 haplotype $(-19 \mathrm{G}>\mathrm{A},+47 \mathrm{~T}>\mathrm{C})$, which was present in the proband, II-5, and her affected sister, II-4, was used as the background sequence. The 79T allele had strong expression of miR-133a-3p with low amounts of miR-133a-5p. When compared with $79 \mathrm{~T}$, the $79 \mathrm{C}$ allele showed no significant change in miR-133a-3p $(P=0.81)$ but there was a mean 3.6 -fold increase in levels of miR-133a-5p $(P=0.003$, Figure 1D). 
Table 1 MiR-1 and miR-133 gene variants identified in familial AF probands

\begin{tabular}{|c|c|c|c|c|c|c|c|c|}
\hline \multirow{2}{*}{ Gene } & \multirow{2}{*}{ Chromosome } & \multirow{2}{*}{$\begin{array}{l}\text { Nucleotide } \\
\text { change }^{a}\end{array}$} & \multirow{2}{*}{$\begin{array}{l}\text { Major/Minor } \\
\text { allele }\end{array}$} & \multicolumn{4}{|c|}{ Minor allele frequency ${ }^{\mathbf{b}}$} & \multirow{2}{*}{$\mathrm{dbSNP} \mathrm{P}^{\mathrm{c}}$} \\
\hline & & & & $\mathrm{AF}$ & Controls & ESP & $1000 \mathrm{G}$ & \\
\hline MIR1-1 & 20 & $-43 C>T$ & $\mathrm{C} / \mathrm{T}$ & 0.004 & NA & - & - & Novel \\
\hline \multirow[t]{2}{*}{ MIR1-2 } & 18 & $+15 A>G$ & $A / G$ & 0.017 & NA & 0.020 & 0.018 & rs9989532 \\
\hline & & $+74 \mathrm{~T}>\mathrm{C}$ & $\mathrm{T} / \mathrm{C}$ & 0.046 & NA & 0.087 & 0.071 & rs78641532 \\
\hline \multirow[t]{6}{*}{ MIR133A2 } & 20 & $-102 G>A$ & $\mathrm{G} / \mathrm{A}$ & 0.142 & 0.122 & - & 0.207 & rs45547937 \\
\hline & & $-82 G>A$ & $\mathrm{G} / \mathrm{A}$ & 0.142 & 0.122 & - & 0.206 & rs13040566 \\
\hline & & $-19 \mathrm{G}>\mathrm{A}$ & $\mathrm{G} / \mathrm{A}$ & 0.142 & 0.122 & 0.229 & 0.207 & rs13040413 \\
\hline & & $79 \mathrm{~T}>\mathrm{C}$ & $\mathrm{T} / \mathrm{C}$ & 0.004 & 0 & - & - & Novel \\
\hline & & $+47 \mathrm{~T}>\mathrm{C}$ & $\mathrm{T} / \mathrm{C}$ & 0.400 & 0.340 & 0.388 & 0.387 & rs6062251 \\
\hline & & $+69 G>A$ & $\mathrm{G} / \mathrm{A}$ & 0.004 & 0.010 & 0.006 & 0.009 & rs149629841 \\
\hline MIR133B & 6 & -25 delA & $A /-$ & 0.083 & NA & 0.075 & 0.074 & rs 142410335 \\
\hline
\end{tabular}

${ }^{a}$ Location of nucleotide changes is shown as (-) downstream or (+) upstream of the miRNA stem-loop sequence (as annotated by miRBase).

${ }^{b} A F$, atrial fibrillation ( $\mathrm{n}=120$, this study cohort); Controls, healthy volunteer subjects $(\mathrm{n}=250$, this study cohort); ESP, NHLBI Exome Sequencing Project (European American subgroup data); 1000G, 1000Genomes Project (European subgroup data). NA, data not available (not tested).

c dbSNP, Database of Single Nucleotide Polymorphisms [http://www.ncbi.nlm.nih.gov/projects/SNP/].

\section{MiR-133a populations in the normal atrium}

To determine the diversity and abundance of miR-133a-3p and $5 \mathrm{p}$ processed species that are normally present in the atrium, small RNA libraries were prepared from human and murine heart tissue samples and were subjected to deep sequencing. A comprehensive list of the sequences and expression levels of all the cardiac miRNA isomiRs identified is provided in the (Additional file 1: Table S1 and Additional file 2: Table S2). In human and murine atrial tissues, miR-133 was the most highly expressed miRNA, comprising approximately $20 \%$ of all miRNA sequences. Analysis of sequencing tags that map to a miR-133a locus showed an extensive range of $5^{\prime}$ and $3^{\prime}$ isomiRs for miR-133a-3p and miR-133a-5p in both human and mouse (Additional file 3: Table S3 and Additional file 4: Table S4). There were two predominant isomiRs processed from the miR-133a $3 \mathrm{p}$ arm in both the human and murine atria (Figures $2 \mathrm{~A}$ and $2 \mathrm{~B}$ ). The sequences for the miR-133a high-abundance isomiRs were identical in the two species. Together, the abundance of these tags represented $>99 \%$ of all tags derived from the miR-133a hairpin. Interestingly, the more abundant sequence in both human and murine samples was not the sequence annotated as the mature form in miRBase, but rather the +1 isomiR. Less than $1 \%$ of all sequences that mapped to the miR-133a hairpin aligned to the $5 \mathrm{p}$ arm. There was a single predominant miR-133a-5p isomiR in human atrium, which started one nt upstream from the murine miRBase entry. There was also only one major murine miR-133a-5p isomiR that had an identical $5^{\prime}$ sequence to the major human miR-133a-5p isomiR.

\section{MiR-133a mRNA target profiles}

We hypothesized that increases in miR-133a-5p associated with the $79 \mathrm{~T}>\mathrm{C}$ MIR133A2 variant could result in selective down-regulation of a distinctive set of target mRNAs. To explore what these cardiac genes might be, we used the seed sequences of the two most abundant human miR-133a-3p isomiRs and the single abundant miR-133a-5p isomiR identified by deep sequencing to look for predicted human mRNA binding sites in TargetScan. For the two abundant human miR-133a-3p isomiRs, the seed sequences TGGTCCC and TTGGTCC had 402 and 502 predicted targets, respectively, of which 247 were in common. Only $26(6 \%)$ of the total 402 TGGTCCC human miR-133a-3p isomiR targets overlapped with the predicted targets for the abundant human miR-133a-5p, and similarly, 30 (6\%) of the total 502 TTGGTCC isomiR targets overlapped with those predicted for human miR-133-5p. Overall, only 44 (7\%) of the total 657 human miR-133a-3p predicted targets from both abundant isomiRs overlapped with the targets predicted for miR-133a-5p (Figure 2C). To compare murine miR-133a-3p and miR-133a-5p targets, we used the two predominant $3 \mathrm{p}$ and single predominant $5 \mathrm{p}$ murine isomiR seed regions to search for predicted mRNA binding sites in TargetScan. Similar to the human data, only a minority of murine miR-133a-3p targets (31 [5\%]) overlapped with $5 \mathrm{p}$ targets (Figure 2D). When all TargetScan-predicted targets were considered, there was substantial concordance across species with 514 (78\%) of human miR-133a-3p targets and 259 (78\%) of human miR-133a-5p targets shared by mouse. We then crossreferenced all the predicted murine targets to a set of mRNAs identified by Liu and colleagues [21] that showed altered expression levels in miR-133a knockout mice. This produced a list of 61 predicted targets for miR-133a-3p and 35 predicted targets for miR-133a-5p, only 4 of which were in common (Figure 2D). We also compared our TargetScan outputs to a list of $1640 \mathrm{miR}-133 \mathrm{a}$ 


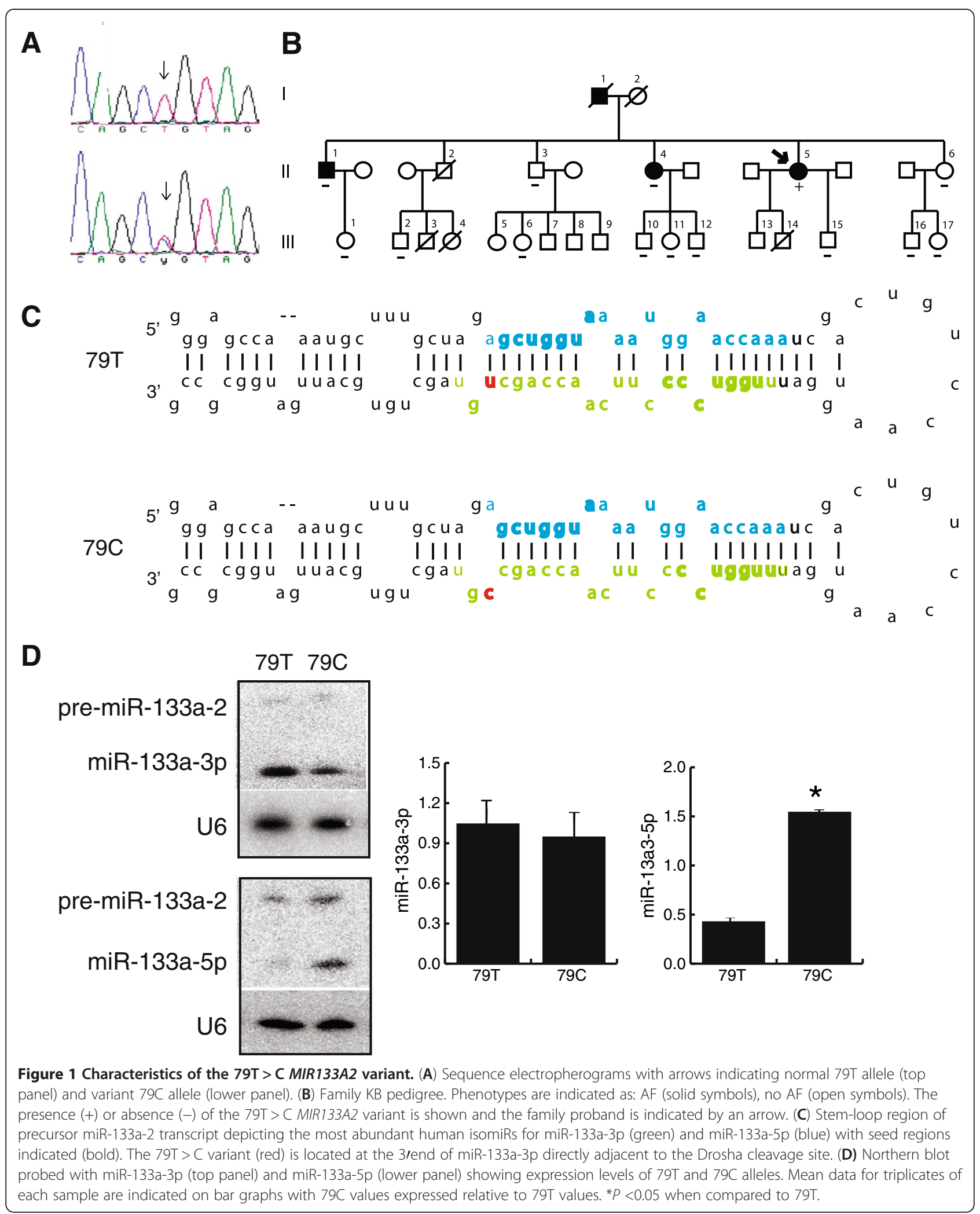




\section{Human}

A

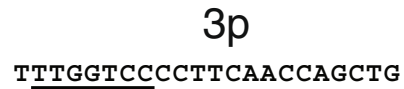

TTGGTCCCCTTCAACCAGCTGT

TTTGGTCCCCTTCAACCAGCTGT

TGGTCCCCTTCAACCAGCTGT

GGTCCCCTTCAACCAGCTGT

ATTTGGTCCCCTTCAACCAGCTGT

$5 p$

(no miRBase sequence)

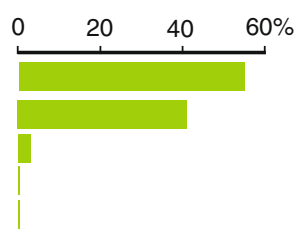

GCTGGTAAAATGGAACCAAAT

GAGCTGGTAAAATGGAACCAAA

TGGTAAAATGGAACCAAATCGA

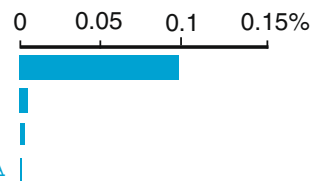

\section{Mouse}

B

$3 p$

TTTGGTCCCCTTCAACCAGCTG

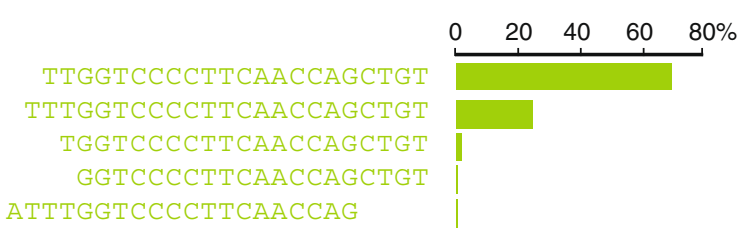

$5 p$

GCTGGTAAAATGGAACCAAAT
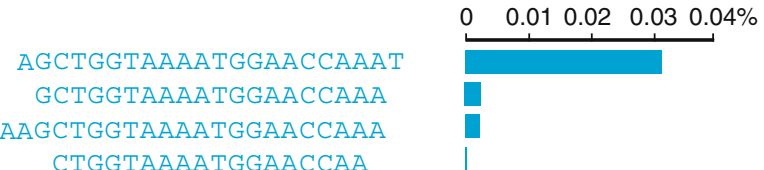

CTGGTAAAATGGAACCAA
C

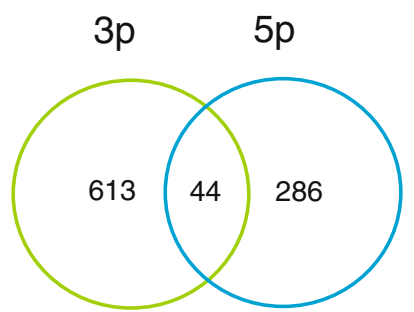

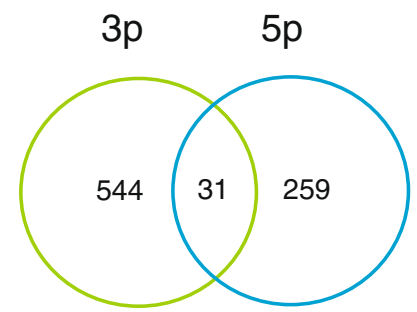

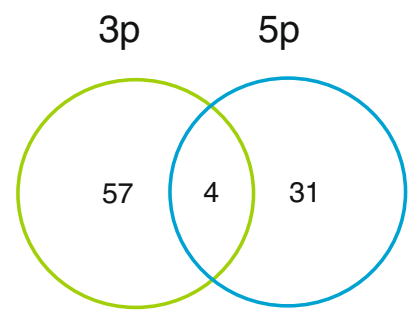

Figure 2 Abundance of miR-133a $\mathbf{5}^{\prime}$ isomiRs in atrial tissue. MiR-133a-3p (green) and miR-133a-5p (blue) isomiRs were evaluated by deep sequencing of human (A) and murine (B) atrial tissues. $5^{\prime}$ isomiRs affect the seed sequences and are considered functionally more important than $3^{\prime}$ isomiRs. The most abundant $5^{\prime}$ isomiRs, with the most frequently-occurring $3^{\prime}$ variant for each of these, are shown. MirBase-annotated miR-133a-3p and $5 p$ sequences are shown in black with 'seed' regions (nt 2-8) underlined. Note that there is no miRBase annotation for human miR-133a-5p. (C) Venn diagram showing numbers of human target mRNAs predicted by TargetScan with the seed regions of the 2 most abundant human miR-133a-3p isomiRs and the single abundant miR-133a-5p isomiR used as inputs. (D) (Left panel) Murine target mRNAs predicted by TargetScan with seed regions of the 2 abundant murine miR-133a-3p isomiRs and single abundant miR-133a-5p isomiRs as inputs. (Right panel) Venn diagram showing numbers of putative miR-133a-3p and miR-133a-5p target mRNAs determined by cross-referencing predicted murine targets with genes demonstrated to be differentially regulated in the hearts of miR-133a knockout mice [22].

targets identified by Matkovich and colleagues in mouse heart using RISC-seq [16]. When the TargetScan and RISC-seq data were compared, there were 168 miR-133a-3p targets when considering both major isomiRs, and 75 miR-133a-5p targets, only 6 of which were common. Matkovich and colleagues reduced their list of 1640 targets to 209 by analyzing transgenic mice that overexpress miR-133a [16]. A comparison of TargetScan targets with this refined list identified 27 miR-133a-3p targets when considering both major isomiRS and 4 miR-133a-5p targets, with no targets in common. These findings collectively suggest that miR-133a isomiRs have distinctive target spectra. In the Ingenuity Knowledge Base, the TargetScan-predicted human miR-133a-3p and 5p target mRNAs are associated with a range of cardiac and extra-cardiac biological functions (Figure 3).

\section{Discussion}

There are two major outcomes of this study. First, we have characterized atrial miR-133a species and find an extraordinary diversity of $5^{\prime}$ and $3^{\prime}$ miR-133a-3p and miR-133a-5p isomiRs, with two major 3p isomiRs and one major $5 \mathrm{p}$ isomiR in both human and mouse. These isomiRs have minimally-overlapping suites of predicted mRNA targets and functions, which suggests that they have distinct biological roles. Second, we report a novel MIR133A2 variant that alters strand abundance during miRNA processing and results in accumulation of miR133a-5p. This variant is one of only a very few functional miRNA gene variants reported to date.

Despite the enormous interest in miRNAs as master regulators of gene expression, the role of genetic variation is incompletely understood. Single nucleotide polymorphisms (SNPs) in predicted miRNA binding 


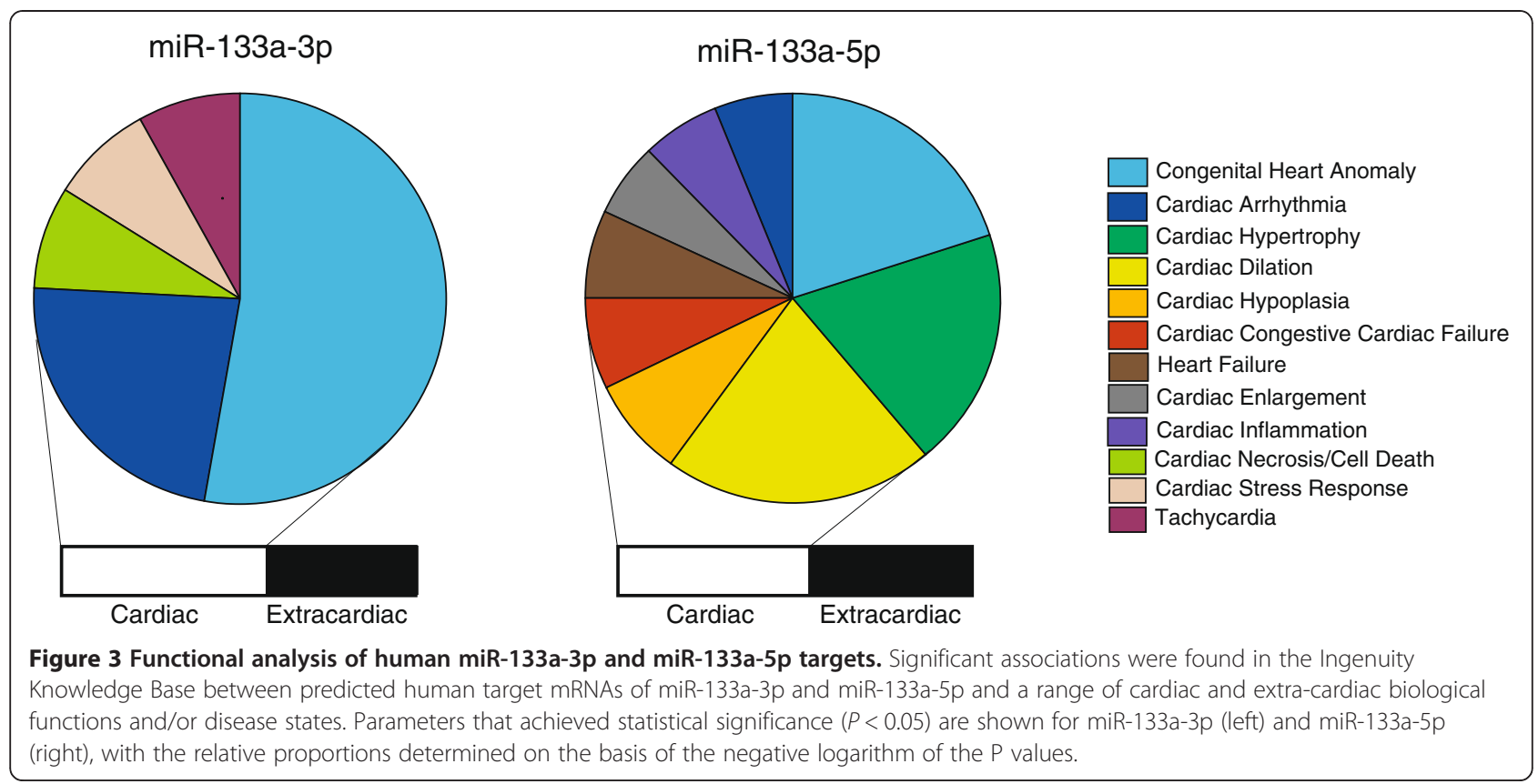

sites on target mRNAs occur frequently and some of these have been associated with phenotypic traits [22-25]. In contrast, SNPs in the miRNA genes themselves are far less common. Several SNPs in pri-miRNA and pre-miRNA have been functional evaluated and shown to result in defective processing and reduced levels of mature miRNA. SNPs are rarely seen $(<1 \%)$ in the seed regions that are crucially required for target recognition [22]. These observations have provided an argument for strong selective constraint and suggest that seed variants would have substantial effects. The discovery of point mutations in the seed region of miR-96 in two families with nonsyndromic progressive hearing loss provided the first example of human Mendelian disease associated with a miRNA gene variant [26]. Functional SNPs can occur in mature miRNAs outside the seed region, as recently demonstrated by the finding of a rare variant at nt 17 of miR-499 [27]. Evaluation of the effects of this variant in transgenic mice showed that it protected against the cardiomyopathy that developed with overexpression of the wildtype form of miR-499. Here we describe a MIR133A2 variant located in the mature miRNA outside the seed region that changes the way that the miRNA duplex is processed.

MiRNA processing is conventionally assumed to yield only one functional mature miRNA with the passenger strand being degraded and hence, functionally irrelevant. It has recently been appreciated however, that passenger forms are not always selected for degradation, rather, that both $3 p$ and $5 p$ strands of the miRNA duplex may be simultaneously present and that their ratio can be regulated in a tissue- or development-specific manner, suggesting that both strands have inherent functional activity [28]. Here we find that both (mature) miR-133a-3p and (passenger) miR-133a-5p are present in the atrium in humans and mice, with miR-133a-5p normally representing $<1 \%$ of all miR-133a species. The main effect of the 79T > C MIR133A2 variant is to alter the relative ratio of miR-133a-3p and $5 p$ strands.

Current models posit that the strand of the miRNA duplex with weaker base-pairing at the $5^{\prime}$ end is preferentially incorporated into RISC [4]. If the conventional murine miR-133a-5p sequence as annotated by miRBase (v18) represented the predominant isomiR in the normal human heart, then the nt corresponding to position 79 would lie just outside the base-paired region of the processed miRNA duplex. However, our deep sequencing data clearly show that the -1 isomiR is the most abundant miR-133a-5p species in the human atrium. Consequently, the $79 \mathrm{~T}>\mathrm{C}$ variant lies within the duplex and would directly prevent base-pairing and weaken thermostability at this site, favoring incorporation of miR-133a-5p into RISC. Additionally the lack of base pairing at position $79 \mathrm{C}$ is likely to enlarge the bulge in the stem-loop structure, a feature known to influence positional cleavage by Dicer and Drosha and result in increased variability of $5^{\prime}$ isomiRs $[8,29]$. The canonical miRNA biosynthesis pathway predicts $3 p$ and $5 p$ miRNAs of $22 \mathrm{nt}$ in length, but deep sequencing studies have revealed a surprising extent of isomiR diversity in most tissues and species [5-8]. These isomiRs may include $5^{\prime}$ or $3^{\prime}$ cleavage variations, and non-templated additions or trimming at the $3^{\prime}$ end. It has been found that the relative abundance and types of isomiRs in specific 
tissues can vary over time and with disease states, suggesting that they have distinctive biological functions $[5,8,30]$.

It is conventionally assumed that the seed regions are the major determinants of target specificity, and thus it is plausible that $5^{\prime}$ isomiR variation can affect mRNA target selection. For example, our group has recently demonstrated that the two most abundant miR-133a isomiRs in murine atrial HL-1 cells have different targeting properties [8]. Given that each isomiR has hundreds of mRNA targets, dynamic fluxes in isomiR characteristics may give rise to an unexpected complexity of miRNA:mRNA interactions and provide a mechanism for intricate regulation of gene expression in specific tissues. To assess the potential impact of the 79T > C MIR133A2 variant, we searched for differences in miR-133a-3p and miR-133a-5p predicted mRNA targets using the respective human and murine seed regions and a set of genes known to be differentially regulated in miR-133a knockout mice [21]. These results showed that only a minority of predicted miR-133a targets were shared, and that most were unique to either $3 p$ or $5 p$ forms. With the 79T > C MIR133A2 variant, no changes in miR-133a-3p target gene expression would be expected. In contrast, the relative increase in miR-133a-5p could have a relatively greater impact and give rise to selective repression of the $5 \mathrm{p}$ suite of targets. It is notable that at least $25 \%$ of the lowest-abundance miR-133a-5p targets include mRNAs involved in regulation of transcription, signaling and membrane transport. Further studies are required to determine whether changes in miR-133a-5p directly alter levels of these critical molecules and have biologically-significant functional effects.

The relationship, if any, between the $79 \mathrm{~T}>\mathrm{C}$ MIR133A2 variant and AF in the Family KB proband is questionable. Although DNA samples were unavailable from the deceased parents, it seems most likely that the $79 \mathrm{~T}>\mathrm{C}$ substitution is a de novo sequence change which was uniquely present in one family member. A causal gene mutation that can account for the familial pattern of AF in this kindred has yet to be identified. The presence of the MIR133A2 variant in the Family KB proband could foreseeably have a modifying effect by altering gene expression profiles in the atrium. A number of factors in addition to genetic variation need to be considered in this individual, including the impact of AF per se and of co-existent risk factors for AF. Altered expression of miR-133 itself has been observed in cardiac tissues from patients with AF $[18,19]$, and conditions that predispose to AF, such as atrial dilation, ventricular hypertrophy, and myocardial ischemia [12,31]. It is notable that individual II-5 had both hypertension and valvular heart disease which independently increase AF risk. A limitation of this study is the lack of atrial tissue samples to document gene expression patterns in 79T and $79 \mathrm{C}$ family members. The collective effects of the multiple variables present in individual II-5 on atrial gene expression and the electrical and structural properties of the atrial wall are difficult to predict and could promote or protect against AF.

\section{Conclusions}

MiRNA duplex processing generates a dominant strand that is incorporated into RISC and a passenger strand that is usually degraded. In the normal human atrium, almost all the miR-133a is comprised of miR-133a-3p with negligible amounts of miR-133a-5p. Although multiple $5^{\prime}$ and $3^{\prime}$ isomiRs are present, there are only 2 major miR-133a-3p isomiRs and one major miR-133a-5p isomiR. These isomiRs have distinctive suites of predicted gene targets suggesting independent biological roles. Very few genetic variants in mature miRNA sequences have been reported and functionally characterized. We have identified a missense MIR133A2 variant that alters miR-133a duplex processing and strand abundance with accumulation of miR-133a-5p in HeLa cells. Further studies to confirm these findings and to document mRNA expression profiles in patient heart tissues are indicated. Despite the importance of miR-133a in atrial biology, miR-133a genetic variants are not a common cause of familial AF.

\section{Methods}

\section{Genetics study subjects}

One hundred and twenty subjects with suspected familial AF were identified from St Vincent's Hospital and by physician referral. Family probands and their relatives were evaluated by medical history and physical examination, 12-lead echocardiography and transthoracic echocardiography. A positive family history was defined as the presence of documented AF in 2 or more firstdegree family members. Two hundred and fifty healthy individuals with no history of cardiovascular disease comprised a control group. All families and control subjects were of Western European background. Genomic DNA was isolated from whole blood samples using the standard salting-out technique. Informed, written consent was obtained from all participants and protocols were approved by the St Vincent's Hospital campus human and animal research ethics committees.

\section{Genetic analysis}

MIR1-1, MIR1-2, MIR133A1, MIR133A2, and MIR133B were re-sequenced in DNA samples from family probands. Primer sequences were designed to amplify regions spanning at least 120 bp upstream and downstream of the stem-loop sequences (as annotated by 
miRBase) of hsa-miR-1-1, hsa-miR-1-2, hsa-miR-133a-1, hsa-miR-133a-2, hsa-miR-133b (miRBase accession numbers MI0000651, MI0000437, MI0000450, MI0000451, MI0000822, respectively). Polymerase chain reaction (PCR) was carried out on genomic DNA using FastStart Taq DNA Polymerase (Roche, Indianapolis, IN, USA) on a Dyad Peltier Thermal Cycler (Bio-Rad Laboratories, Hercules, CA, USA) with the primer pairs shown in Table 2, designed using the programs Primer3Plus and Amplify version 3.1.4 (University of Wisconsin, Madison, WI, USA). Cycling conditions for MIR1-1, MIR133A2 and $M I R 133 B$ are as follows: $94^{\circ} \mathrm{C}(3 \mathrm{~min})$, then $35 \mathrm{cy}-$ cles of $94^{\circ} \mathrm{C}(20 \mathrm{~s}), 55^{\circ} \mathrm{C}(30 \mathrm{~s}), 72^{\circ} \mathrm{C}(60 \mathrm{~s})$ and finally $72^{\circ} \mathrm{C}$ (8 $\left.\mathrm{min}\right)$. MIR1-2 and MIR133A1 were amplified using the "Slowdown PCR" method. Amplicons were purified with ExoSAP-IT enzyme (GE Healthcare, Chalfont St Giles, Buckinghamshire, UK), sequenced using Big Dye Terminator (version 3.1, Applied Biosystems, Foster City, CA, USA) and analyzed on an ABI PRISM 3700 DNA Analyzer (The Ramaciotti Centre for Gene Function Analysis, UNSW, Sydney, New South Wales, Australia). Viewing and analysis of the data was carried out using the DNASTAR software package (Madison, WI, USA). DNA sequence variants were validated by re-sequencing independent PCR-generated amplicons from probands in both directions. Sequence variants identified in family probands were subsequently evaluated in affected and unaffected family members and in the cohort of healthy control subjects.

\section{Plasmid constructs and cell transfections}

A 272bp fragment containing the precursor sequence of mature miR-133a-2 with 79T and 79C alleles was amplified from genomic DNA using gene-specific primers containing BamHI and HindIII restriction enzyme sites (underlined): forward: $5^{\prime}$ - ggggcggccgcggatccatctccatcgg gactgc $-3^{\prime}$, reverse: $5^{\prime}$ - gggaagcttggcactcagggcttcactta $-3^{\prime}$.

Table 2 Primer sequences used for PCR amplification and sequencing

\begin{tabular}{llll}
\hline $\begin{array}{l}\text { Primer } \\
\text { name }\end{array}$ & Primer sequence & $\begin{array}{l}\text { Amplicon } \\
\text { size (bp) }\end{array}$ & $\begin{array}{l}\text { Sequencing } \\
\text { primer }\end{array}$ \\
\hline mir-1-1F & $5^{\prime}$ - gagatggattcagggatgga -3' & 494 & mir-1-1R \\
mir-1-1R & $5^{\prime}$-acctgctgacacaggcaaag -3' & & \\
mir-1-2F & $5^{\prime}$-ggaaccattaatgccatgct -3' & 467 & mir-1-2F \\
mir-1-2R & $5^{\prime}$ - tgaaatctacttcactggatcttctt -3' & \\
mir-133a-1F & $5^{\prime}$ - tttaaaccattaagcgcagga -3' & 455 & mir-133a-1F \\
mir-133a-1R & $5^{\prime}$ - ttgaaatccttaagtcatccataca -3' & mir-133a-2R \\
mir-133a-2F & $5^{\prime}$ - ctgcagagcttgagggaaac -3' & 466 & \\
mir-133a-2R & $5^{\prime}$ - caaggaggaacaagcaggag -3' & & \\
mir-133bF & $5^{\prime}$ - agtcatgcaacatgaaatacaaa -3' & 500 & mir-133bR \\
mir-133bR & $5^{\prime}$ - gagtgcaaaggcacagaaca -3' & & \\
\hline
\end{tabular}

Amplification was carried out using FastStart Taq DNA Polymerase with the same cycling condictions as described for MIR1-1, MIR133A2 and MIR133B above. PCR amplicons were purified using the QIAquick Gel Extraction Kit (Qiagen, Hildem, Germany) and cloned into the $\mathrm{pGEM}^{\circledR}-\mathrm{T}$ Easy Vector system (Promega, Fitchburg, WI, USA) according to manufacturer's instructions. Clones were digested with BamHI and HindIII and ligated to BamHI- and HindIII-digested pSilencer ${ }^{\mathrm{TM}}$ puro 4.1-CMV vector (Applied Biosystems). All plasmid constructs were confirmed by sequencing.

HeLa cells (ATCC, Manassas, VA, USA) were maintained in Dulbecco's modified eagle medium (DMEM) with 5\% fetal calf serum, supplemented with glutamine and penicillin-streptomycin. Cells were seeded in a 6-well plate 8 hours before transfection. Transfections were performed in duplicate at $\sim 70 \%$ confluency by adding $1.5 \mathrm{~mL}$ of DMEM and $1 \mathrm{~mL}$ of transfection solution per well. The $1 \mathrm{~mL}$ transfection solution contained Opti-MEM ${ }^{\circledR}$, 150ng of $79 \mathrm{~T}$ or $79 \mathrm{C}$ constructs and $5 \mathrm{uL}$ of Lipofectamine 2000 reagent (Invitrogen, Carlsbad, CA, USA), which was incubated for 20 minutes at room temperature prior to transfection.

\section{RNA analysis}

HeLa cells (ATCC) were harvested after 24 hours and total RNA was extracted using TRIzol $^{\circledR}$ (Invitrogen). Total RNA (5ug) was separated on $5 \%$ stacking/12\% resolving polyacrylamide gels, transferred electrophoretically to Gene Screen Plus ${ }^{\circledR}$ Hybridization Transfer Membranes (Perkin Elmer, Waltham, MA, USA). Separated RNA was fixed on the membranes by $\sim 20 \mathrm{sec}$ UV crosslinking on a UV Stratalinker 2400 (Stratagene, Santa Clara, Ca, USA). Northern blotting was performed as described [32]. DNA oligonucleotide probes $\left(5^{\prime}-\right.$ taca gctggttgaaggggaccaaa $-3^{\prime}, 5^{\prime}$ - gatttggttccattttaccagct $-3^{\prime}, 5^{\prime}-$ tgtgctgccgaagcaagcac $-3^{\prime}$ ) complementary to mature miR-133a (miR-133a-3p), passenger miR-133a (miR-133a-5p) and U6 sequences, respectively, were end-labeled with ${ }^{32} \mathrm{P}$ using T4 Polynucleotide Kinase (New England Biolabs, Ipswich, MA, USA) and purified by Microspin G-25 columns (GE Healthcare) according to manufacturer's instructions. A single probe was incubated with the membrane in hybridization buffer $(250 \mathrm{mM} \mathrm{NaPO}, 7 \%$ SDS, $\mathrm{pH} 7$ ) at $42^{\circ} \mathrm{C}$ overnight. After hybridization, membranes were washed 3 times with $2 x S S C(1.753 \%$ $\left.\mathrm{NaCl}, 0.882 \% \mathrm{Na}_{3} \mathrm{C}_{6} \mathrm{H}_{5} \mathrm{O}_{7}\right)$ at $42^{\circ} \mathrm{C}$, and exposed overnight. Phosphorimaging was performed on a Fuji imager (FLA-5100, Fujifilm, Minato-ku, Tokyo, Japan). Hybridization signals were quantified using Multi Gauge v2.3 software (Fujifilm, Minato-ku, Tokyo, Japan) and the values measured for each probe from each blot were scaled to the average signal. The scaled miR-133a values were then normalized to the scaled U6 values to adjust for 
any loading bias. Statistical analyses were performed using the Mann-Whitney $U$ test with a $P$ value $<0.05$ deemed significant.

\section{Deep sequencing}

For deep sequencing of atrial myocardium, total RNA was extracted using the miRNeasy ${ }^{\circledR}$ Kit (QIAGEN, Hildem, Germany) from right atrial appendage samples obtained from two males aged 62 and 64 years with no history of AF who underwent coronary artery bypass graft surgery. Atrial tissue was also obtained from the hearts of 3 wildtype mice aged 4-6 weeks. Small RNA libraries were created using NEBNext ${ }^{\circledR}$ small RNA library preparation kit (New England Biolabs, Ipswich, MA, USA) and sequenced on a $5500 \mathrm{SOLiD}^{\mathrm{TM}}$ platform (Applied Biosystems). Sequencing data were mapped using Lifescope software (Applied Biosystems). Processed miRNA counts were initially collated from all tags that aligned within 3 nt of the miRBase defined 5/ starting position. Tags that mapped to miRBAse v18 can be accessed via a "miRDSPRing" document (D. Humphreys, manuscript in preparation) and are available in the Supporting Information (Tables S1 and S2).

\section{MiRNA target predictions}

Gene targets for human and murine miR133a were predicted using TargetScan Custom (v5.2) [33] with the "seeds" (nt 2-8) of the most abundant miR-133a-3p and miR-133a-5p isomiRs that were identified via deep sequencing used as inputs. The newer version of TargetsScan (v6.2) and a range of other commonly-used publicallyavailable target prediction programs (DIANA-microT, Microinspector, microRNA.org, MirTarget2, PicTar, PITA, RNA22, RNAhybrid) were unable to be utilized because human and/or murine miR-133a-5p sequences were unable to be inputted and/or analyzed. Predicted gene targets of murine miR-133a were matched against mRNAs that in which expression levels were altered by at least 1.5 fold in microarray data from miR-133a knockout mice [21]. The TargetScan outputs for human miR-133a-3p and miR-133a $-5 p$ were imported into Ingenuity Pathway Analysis software (Ingenuity ${ }^{\circledR}$ Systems, www.ingenuity.com) for identification of biological pathways in the heart that might be differentially regulated (Additional file 5: Table S5).

\section{Additional files}

Additional file 1: Table S1. Alignment of tags derived from mouse atria with miRNA hairpins as listed in miRBase version 18.

Additional file 2: Table S2. Alignment of tags derived from human right atrial appendage with miRNA hairpins as listed in miRBase version 18.

Additional file 3: Table S3. Sequences and abundance of different $5^{\prime}$ and $3^{\prime}$ murine miR-133a isomiRs identified by sequencing of murine atria.
Additional file 4: Table S4. Sequences and abundance of different $5^{\prime}$ and $3^{\prime}$ human miR-133a isomiRs identified by sequencing of human atria.

Additional file 5: Table S5. TargetScan outputs for human miR-133a-3p and miR-133a-5p (from Figure 2C) that were used as inputs for Ingenuity Pathway Analysis.

\section{Abbreviations}

AF: Atrial fibrillation; DMEM: Dulbecco's modified eagle medium; miRNA: microRNA; nt: Nucleotide; PCR: Polymerase chain reaction; premiRNA: Precursor miRNA; pri-miRNA: Primary miRNA; RISC: RNA-induced silencing complex.

\section{Competing interests}

The authors declare that they have no competing interests.

\section{Authors' contributions}

MO performed the genetics studies, plasmid construct preparations, cell transfections, RNA analyses, miRNA target predictions and drafted parts of the manuscript. DTH carried out RNA analyses and performed deep sequencing experiments and analysis. EA performed clinical evaluation of family members. TP participated in the design of the study and provided intellectual input into data analysis. DF conceived the study, participated in its design and drafted the manuscript. All authors read and approved the final manuscript.

\section{Acknowledgements}

We thank collaborating physicians for proband referrals; Poonam Zodgekar and Kathryn Stockhammer, for family member recruitment; Gunjan Trivedi and Lena Soka, for laboratory assistance; Kartik Ramesh, Keng Tan, Li Sze Yeo, and Khumud Dhital for collection of atrial tissue samples; Cath Suter, Carly Hynes, and Hardip Patel, for assistance with deep sequencing and miRNA analysis.

\section{Author details}

${ }^{1}$ Molecular Cardiology Division, Victor Chang Cardiac Research Institute, Darlinghurst, New South Wales, Australia. ${ }^{2}$ Molecular Genetics Division, Victor Chang Cardiac Research Institute, Darlinghurst, New South Wales, Australia. ${ }^{3}$ Faculty of Medicine, University of New South Wales, Kensington, New South Wales, Australia. ${ }^{4}$ Capital Cardiac Centre, Garran, Australian Capital Territory, Australia. ${ }^{5}$ Genome Biology Department, The John Curtin School of Medical Research, The Australian National University, Canberra, Australian Capital Territory, Australia. ${ }^{6}$ Cardiology Department, St Vincent's Hospital,

Darlinghurst, New South Wales, Australia.

Received: 28 August 2012 Accepted: 27 February 2013 Published: 6 March 2013

\section{References}

1. Fabian MR, Sonenberg N, Filipowicz W: Regulation of mRNA translation and stability by microRNAs. Annu Rev Biochem 2010, 79:351-379.

2. Kozomara A, Griffiths-Jones S: miRBase: integrating microRNA annotation and deep-sequencing data. Nucleic Acids Res 2011, 39(Suppl 1):D152-D157.

3. Mendell JT, Olson EN: MicroRNAs in stress signaling and human disease. Cell 2012, 148:1172-1187.

4. Winter J, Jung S, Keller S, Gregory RI, Diederichs S: Many roads to maturity: microRNA biogenesis pathways and their regulation. Nat Cell Biol 2009, 11:228-234

5. Fernandez-Valverde SL, Taft RJ, Mattick JS: Dynamic isomiR regulation in Drosophila development. RNA 2010, 16:1881-1888.

6. Lee LW, Zhang S, Etheridge A, Ma L, Martin D, Galas D, Wang K: Complexity of the microRNA repertoire revealed by next-generation sequencing. RNA 2010, 16:2170-2180.

7. Cloonan N, Wani S, Xu Q, Gu J, Lea K, Heater S, Barbacioru C, Steptoe AL, Martin HC, Nourbaksh E, Krishnan K, Gardiner B, Wang X, Nones K, Steen JA, Matigian NA, Wood DL, Kassahn KS, Waddell N, Shepherd J, Lee C, Ichikawa J, McKernan K, Bramlett K, Kuersten S, Grimmond SM: MicroRNAs and their isomiRs function cooperatively to target common biological pathways. Genome Biol 2011, 12:R126.

8. Humphreys DT, Hynes CJ, Patel HR, Wei GH, Cannon L, Fatkin D, Suter CM, Clancy JL, Preiss T: Complexity of murine cardiomyocyte miRNA 
biogenesis, sequence variant expression and function. PLoS One 2012, 7:e30933.

9. Liang $Y$, Ridzon D, Wong $L$, Chen C: Characterization of microRNA expression in normal human tissues. BMC Genomics 2007, 8:166.

10. Luo X, Zhang H, Xiao J, Wang Z: Regulation of human cardiac ion channel genes by microRNAs: theoretical perspective and pathophysiological implications. Cell Physiol Biochem 2010, 25:571-586.

11. Zhao Y, Ransom JF, Li A, Vedantham V, Von Drehle M, Muth AN, Tsuchihashi T, McManus MT, Schwartz RJ, Srivastava D: Dysregulation of cardiogenesis, cardiac conduction, and cell cycle in mice lacking miRNA-1-2. Cell 2007 129:303-317

12. Care A, Catalucci D, Felicetti F, Bonci D, Addario A, Gallo P, Bang ML Segnalini P, Gu Y, Dalton ND, Elia L, Latronico MV, Høydal M, Autore C, Russo MA, Dorn GW II, Ellingsen O, Ruiz-Lozano P, Peterson KL, Croce CM, Peschle C, Condorelli G: MicroRNA-133 controls cardiac hypertrophy. Nat Med 2007, 13:613-618.

13. Duisters RF, Tijsen AJ, Schroen B, Leenders JJ, Lentink V, van der Made I, Herias V, Van Leeuwen RE, Schellings MW, Barenbrug F, Maessen JG, Heymans S, Pinto YM, Creemers EE: MiR-133 and miR-30 regulate connective tissue growth factor: implications for a role of microRNAs in myocardial matrix remodeling. Circ Res 2009, 104:170-178.

14. Matkovich SJ, Wang W, Tu Y, Eschenbacher WH, Dorn LE, Condorelli G, Diwan A, Nerboone JM, Dorn GW II: MicroRNA-133a protects against myocardial fibrosis and modulates electrical repolarization without affecting hypertrophy in pressure-overloaded adult hearts. Circ Res 2010, 106:166-175.

15. Belevych AE, Sansom SE, Terentyeva R, Ho HT, Nishijima Y, Martin MM, Jindal HK, Rochira JA, Kunitomo Y, Abdellatif M, Carnes CA, Elton TS, Györke S, Terentyev D: MicroRNA-1 and-133 increase arrhythmogenesis in heart failure by dissociating phosphatase activity from RyR2 complex. PLOS One 2011, 6:e28324.

16. Matkovich SJ, Van Booven DJ, Eschenbacher WH, Dorn GW: RISC RNA sequencing for context-specific identification of in vivo microRNA targets. Circ Res 2011, 108:18-26.

17. Girmatsion Z, Biliczki P, Bonauer A, Wimmer-Greinecker G, Scherer M, Moritz A, Bukowska A, Goette A, Nattel S, Hohnloser SH, Ehrlich JR: Changes in microRNA-1 expression and IK1 up-regulation in human atrial fibrillation. Heart Rhythm 2009, 6:1802-1809.

18. Xiao J, Liang D, Zhang Y, Liu Y, Zhang H, Liu Y, Li L, Liang X, Sun Y, Chen $\mathrm{YH}$ : MicroRNA expression signature in atrial fibrillation with mitral stenosis. Physiol Genomics 2011, 43:655-664.

19. Cooley N, Cowley MJ, Lin RC, Marasco S, Wong C, Kaye DM, Dart AM, Woodcock EA: Influence of atrial fibrillation on microRNA expression profiles in left and right atria from patients with valvular heart disease. Physiol Genomics 2012, 44:211-219.

20. De Mena L, Coto E, Cardo LF, Diaz M, Blazquez M, Ribacoba R, Salvador C, Pastor P, Samaranch L, Moris G, Menéndez M, Corao Al, Alvarez V: Analysis of the Micro-RNA-133 and PITX3 genes in Parkinson's disease. Am J Med Genet 2010, 153B:1234-1239.

21. Liu N, Bezprozvannaya S, Williams AH, Qi X, Richardson JA, Bassel-Duby R, Olson EN: MicroRNA-133a regulates cardiomyocyte proliferation and suppresses smooth muscle gene expression in the heart. Genes Dev 2008, 22:3242-3254

22. Saunders MA, Liang H, Li WH: Human polymorphism at microRNAs and microRNA target sites. Proc Natl Acad Sci 2007, 104:3300-3305.

23. Borel C, Antonarakis SE: Functional genetic variation of human miRNAs and phenotypic consequences. Mamm Genome 2008, 19:503-509.

24. Kim J, Bartel DP: Allelic imbalance sequencing reveals that singlenucleotide polymorphisms frequently alter microRNA-directed repression. Nat Biotechnol 2009, 27:472-477.

25. Ryan BM, Robles Al, Harris CC: Genetic variation in microRNA networks: the implications for cancer research. Nat Rev Cancer 2010, 10:389-402.

26. Mencia A, Modamio-Hoybjor S, Redshaw N, Morin M, Mayo-Merino F, Olavarrieta L, Aguirre LA, Del Castillo I, Steel KP, Dalmay T, Moreno F, Moreno-Pelayo MA: Mutations in the seed region of human miR-96 are responsible for nonsyndromic progressive hearing loss. Nat Genet 2009, 41:609-613.

27. Dorn GW II, Matkovich SJ, Eschenbacher WH, Zhang Y: A human 3' miR499 mutation alters cardiac mRNA targeting and function. Circ Res 2012 110:958-967.
28. Yang JS, Phillips MD, Betel D, Mu P, Ventura A, Siepel AC, Chen KC, Lai EC: Widespread regulatory activity of vertebrate microRNA* species. RNA 2011, 17:312-326.

29. Starega-Roslan J, Krol J, Koscianska E, Kozlowski P, Szlachcic WJ, Sobczak K Krzyzosiak WJ: Structural basis of miRNA length variety. Nucleic Acids Res 2011, 39:257-268.

30. Marti E, Pantano L, Banez-Coronel M, Llorens F, Minones-Moyano E, Porta S, Sumoy L, Ferrer I, Estivill X: A myriad of miRNA variants in control and Huntington's disease brain regions detected by massively parallel sequencing. Nucleic Acids Res 2010, 38:7219-7235.

31. Bostjancic E, Zidar N, Stajer D, Glavac D: MicroRNAs miR-1, miR-133a, miR$133 \mathrm{~b}$, and miR-208 are dysregulated in human myocardial infarction. Cardiology 2010, 115:163-169.

32. Beilharz TH, Humphreys DT, Clancy JL, Thermann R, Martin DI, Hentze MW Preiss T: MicroRNA-mediated messenger RNA deadenylation contributes to translational repression in mammalian cells. PLoS One 2009, 4:e6783.

33. Lewis BP, Burge CB, Bartel DP: Conserved seed pairing, often flanked by adenosines, indicates that thousands of human genes are microRNA targets. Cell 2005, 120:15-20.

doi:10.1186/1471-2156-14-18

Cite this article as: Ohanian et al:: A heterozygous variant in the human cardiac miR-133 gene, MIR133A2, alters miRNA duplex processing and strand abundance. BMC Genetics 2013 14:18.

\section{Submit your next manuscript to BioMed Central and take full advantage of:}

- Convenient online submission

- Thorough peer review

- No space constraints or color figure charges

- Immediate publication on acceptance

- Inclusion in PubMed, CAS, Scopus and Google Scholar

- Research which is freely available for redistribution 